\title{
Successful treatment of acute respiratory distress syndrome from hydrofluoric acid inhalation
}

\begin{abstract}
Hydrofluoric acid (HF) is an industrial chemical widely used in many industries, including industries of the aluminum, steel, fertilizer, glass, ceramic, and brick. In a variety of settings, HF is a pollutant usually created in an industrial process. ${ }^{1}$ Studies showed that concentrated HF contamination may cause a fatal outcome even if small areas of body surface are exposed., ${ }^{2,3}$ The case reported here is acute respiratory distress syndrome (ARDS) due to accidental inhalation of HF. In cases of refractory hypoxemia, extracorporeal membrane oxygenation (ECMO) is becoming an acceptable rescue therapy. We believe severe hypoxemic respiratory failure as a result of ARDS caused by inhalation of HF in which venovenous-ECMO (VV-ECMO) was used successfully as a bridge to lung recovery has not been previous reported.
\end{abstract}

Keywords: hydrofluoric acid, inhalation, ARDS, ECMO

\author{
Volume 2 Issue 6 - 2017
}

\author{
Liu Xiao lei, ${ }^{2}$ Yang Bao hong,' Liu Ting, ' Xu \\ Qian,' Pan Xiangpo, ${ }^{3}$ Sun Kaiyu² \\ 'Department of Oncology, Weifang People's Hospital, China \\ 2Weifang medical university, China \\ ${ }^{3}$ Department of Clinical laboratory, Weifang People's Hospital, \\ China
} Correspondence: Kaiyu Sun, Weifang medical university,
Weifang Shandong 26104I, PR China,

Email jiangbiao40I2@|26.com

Received: April 25, 2017| Published: May 01, 2017
Abbreviations: HF, hydrofluoric acid; ARDS, acute respiratory distress syndrome; ECMO, extracorporeal membrane oxygenation; VV-EMCO, venovenous-ECMO

\section{Introduction}

A 38year-old Chinese male mechanist who suffered an accidental inhalation of HF from a broken transmission pipe during his working in an aluminum industry started coughing and hemoptysis after approximately 2 hours HF exposure. And he was immediately sent to the local emergency department. The initial chest CT scan showed there were severe patchy infiltrates throughout both lungs of the patient's as showed in Figure 1. The patient was transferred to the intensive care unit for definitive management after the quick medical laboratory examination. The inquiry of the patient showed that he was previous healthy without any systemic illnesses or drug allergies, no history of smoking or regular alcohol consumption. Physical examination revealed a body temperature of $37^{\circ} \mathrm{C}$, a pulse of 120 beats per minute, and a blood pressure of $100 / 60 \mathrm{mmHg}$. Examination of the lungs found decreased breath sounds with occasional wheezes and rales. The electrocardiogram and echocardiogram did not reveal any abnormalities. Chemistry laboratory test showed a red blood cell count of $4.34 \times 10^{\wedge} 12 / \mathrm{L}$, a white blood cell count of $25.96 \times 10^{\wedge} 9 / \mathrm{L}$ (granulocytes $92.5 \%$ ) and a platelet count of $120 \times 10^{\wedge}$ 9/L. The serum level of creatinine was 79umol/1 (reference range: 45-116umol/1), blood urea nitrogen $8.7 \mathrm{mmol} / 1$ (range: $2.7-8.5 \mathrm{mmol} / \mathrm{l}$ ), sodium $142 \mathrm{mmol} / \mathrm{L}$, calcium $1.93 \mathrm{mmol} / \mathrm{L}$ (range: $2.10-2.70 \mathrm{mmol} / \mathrm{L}$ ), magnesium $0.85 \mathrm{mmol} / \mathrm{L}$ (range: $0.72-1.21 \mathrm{mmol} / \mathrm{L}$ ), potassium $4.99 \mathrm{mmol} / \mathrm{L}$, glucose $7.7 \mathrm{mmol} / 1$ (range: $3.9-6.1 \mathrm{mmol} / 1$ ), cardiac isoenzyme of creatine kinase 18U/L (range: $0-20 \mathrm{U} / \mathrm{L}$ ), and creatine kinase 878U/L (range: 38-174U/L). Initial laboratories showed hypocalcemia, the blood gas $(\mathrm{ABG})$ revealed a metabolic acidosis $\left(\mathrm{pH} 7.32\right.$, base deficit $\left.9, \mathrm{HCO}_{3} 16\right)$ and a $\mathrm{PO} 2 / \mathrm{FiO} 2$ ratio 90 , which is consistent with adult respiratory distress syndrome (ARDS).

A chest X-ray was performed in our unit immediately after arrival, and the chest x-ray showed severe patchy infiltrates throughout both lungs as in Figure 2. Besides critical care and ventilatory support, we started the treatment with nebulized calcium gluconate $(2.5 \%)$ for the first 3hours continuously and then every 4hours. We serially monitored electrolytes every 8 hours. After 48 hours, the patient did not have significant calcium requirements and the nebulized calcium gluconate was discontinued. His initial EKG was unremarkable, subsequent EKGs performed after his admission did not reveal any abnormalities.

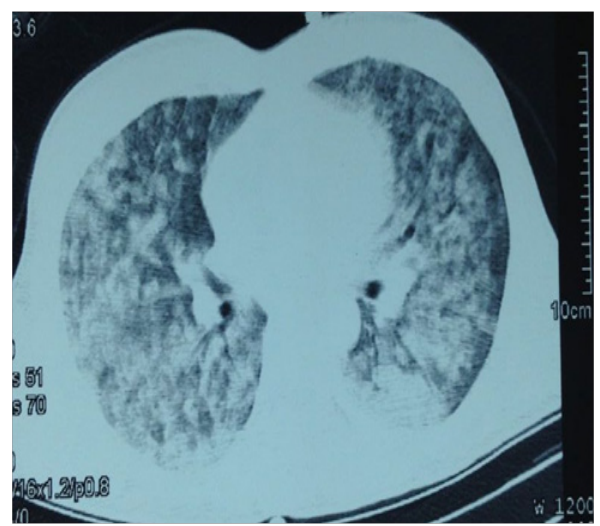

Figure I Initial chest CT scan showing severe patchy infiltrates throughout both lungs.

Considering the patients temperature was increasing and WBC rising, therapy with ampicillin was begun empirically and was later changed to piperacillin when cultures of the sputum grew coagulasepositive sat resistant to ampicillin. Blood cultures grew no organisms.

In the first 48hours, Ventilatory support were made and adjusted occasionally based on arterial blood gases and oxygen saturation. By hospital day 3, the patient's pulmonary function was deteriorating and the $\mathrm{PaO} 2 / \mathrm{FIO} 2$ ratio is only $59 \mathrm{mmHg}$ while on a conventional ventilator with synchronized intermittent mandatory ventilation plus pressure support. We decided to initiate extracorporeal membrane oxygenation (ECMO).

Appropriate consent for initiation of VV-ECMO was obtained and 
the right internal jugular vein was cannulated with a $27 \mathrm{~F}$ Avalon Elite cannula (Maquet Cardiohelp HLS device) within 2hours. Initial VVECMO ows were $3 \mathrm{~L} / \mathrm{min}$ with a sweep ow of $2.5 \mathrm{~L} / \mathrm{min}$ at $100 \% \mathrm{FiO} 2$. Settings were subsequently titrated to clinical status. After VV-ECMO initiation, the patient was placed on pressure control ventilation with $15 \mathrm{~cm} \mathrm{H}_{2} \mathrm{O}$ of PEEP and inspiratory pressure of $25 \mathrm{~cm} \mathrm{H}_{2} \mathrm{O}$.

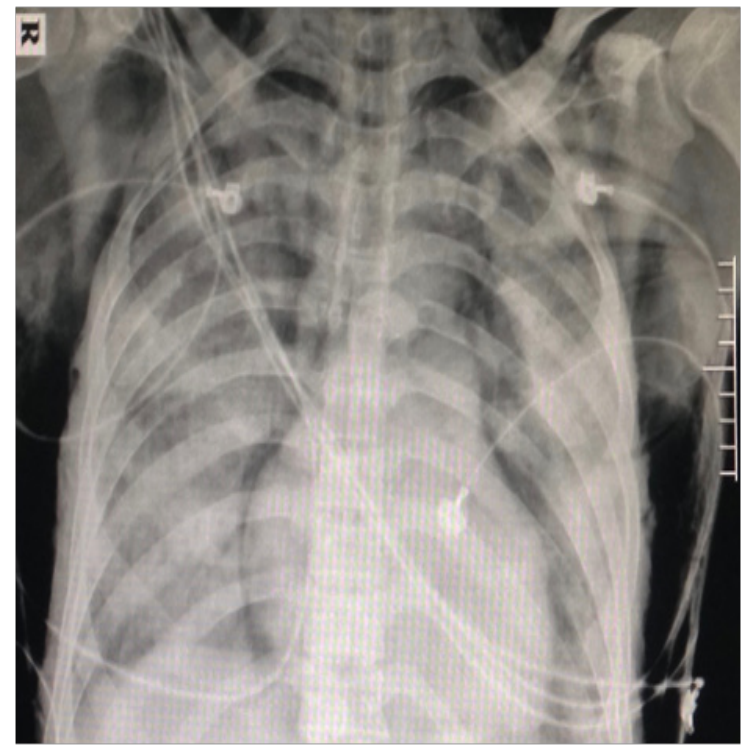

Figure 2 Chest X-ray identified bilateral patchy infiltrates.

Four days after supportive therapy, the patient was weaned successfully from VV-ECMO support. Chest X-ray scan showed a resolving ARDS-type pattern (Figure 3). By hospital day 10, the patient was transferred to the ward, he continued to need ventilatory support and subsequently underwent tracheostomy placement. After 4weeks on the ventilator, ventilatory support was withdrawn considering he tolerated breathing via his tracheostomy for more than 24hours. On hospital day 37, he was discharged from the hospital with no infiltrates in the lungs (Figure 4).

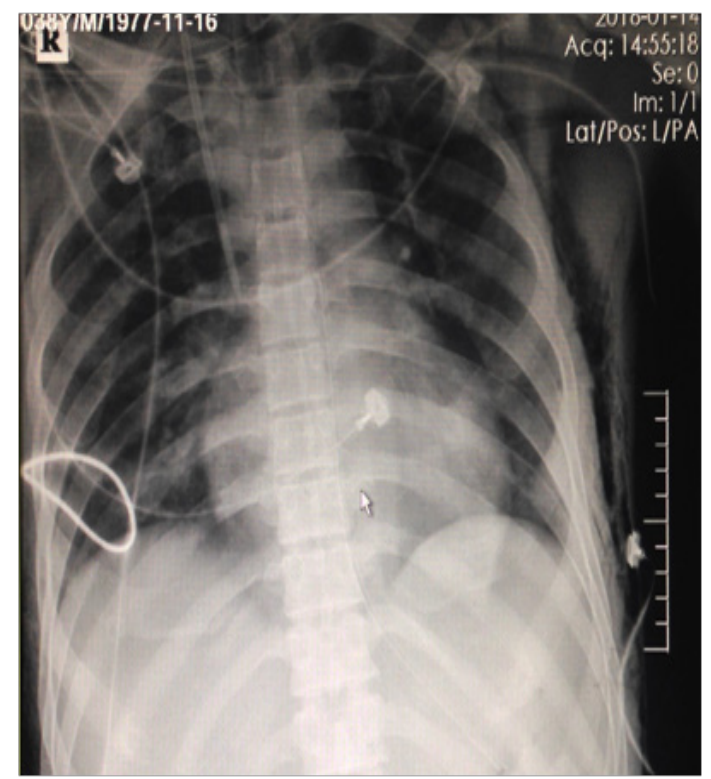

Figure 3 Chest $X$-ray scan showing a resolving ARDS-type pattern after ECMO support.

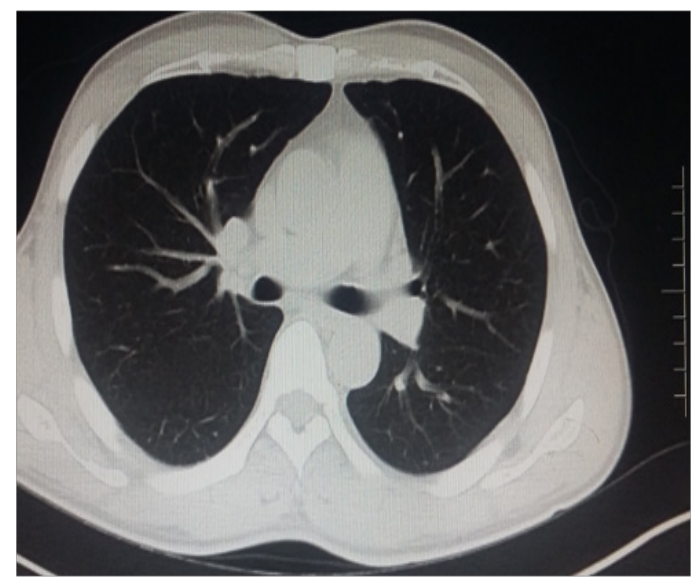

Figure 4 Chest CT scan showing no infiltrates in the lungs when he was discharged.

\section{Discussion}

After inhalation of $\mathrm{HF}$, the highly reactive fluoride ion forms complexes mainly with calcium which can result in severe hypocalcemia, leading to myocardial irritability or dysrhythmias. ${ }^{4}$ Most published case reports-showed that exposure to HF producing hypocalcemia and hypomagnesemia, usually accompanied by hyperkalemia. ${ }^{5}$ The patient in this case report presented with hypocalcemia, but no hypomagnesemia or hypokalemia, which is unusual.

Calcium gluconate was widely used in cutaneous HF exposure. ${ }^{6}$ However, only a few case reports showing the use of calcium gluconate in HF inhalational exposures. ${ }^{7}$ Further clinical studies are necessary.

Acute lung injury resulting in noncardiogenic pulmonary edema after exposure to HF has not previous been reported. When HF was exposed to the upper airways of the rodent models, HF would enter into the systemic circulation rapidly. A very small quantity of HF gas exposure always causing serious problem. The research showed that HF delivered to the lower airways directly caused significant pulmonary hemorrhage and edema. ${ }^{8}$ The development of syndromes have been consistent with studies done in human volunteers. ${ }^{9}$

After failure of conventional modes of ventilation in patients with inhalational injury, the decision to initiate ECMO for this patient was influenced by the recent evidences of ARDS management including early application of ECMO..$^{10-12}$ In the CESAR trial, indications for initiating ECLS were adult patients (18-65years) who had severe but potentially reversible respiratory failure and uncompensated hypercapnea with a $\mathrm{pH} 7.20$ or a Murray score 3.0. The patient we reported here met the criteria outlined by the CESAR trial (38years, $\mathrm{PaO} 2 / \mathrm{FiO} 2$ ratio 59 despite maximal conventional therapy, and Murray score 3.5).

\section{Conclusion}

This case highlights the complexity of treating an inhalation injury from HF. This patient survived, despite the fact that ARDS caused by severe inhalation injuries of HF have been fatal, VV-ECMO should be considered when conventional treatments fail in treating refractory hypoxemia. 


\section{Acknowledgements}

None.

\section{Conflict of interest}

Author declares that there is no conflict of interest.

\section{References}

1. Kirkpatrick JJ, Enion DS, Burd DA. Hydrofluoric acid burns: a review. Burns. 1995;21(7):483-493.

2. Tepperman PB. Fatality due to acute systemic fluoride poisoning following a hydrofluoric acid skin burn. J Occup Med. 1980;22(10):691-692.

3. Meldrum M. Toxicology of hydrogen fluoride in relation to major accident hazards. Regul Toxicol Pharmacol. 1999;30(2):110-116.

4. Greco RJ, Hartford CE, Haith LR, et al. Hydrofluoric acid induced hypocalcemia. J Trauma. 1988;28(11):1593-1596.

5. Dunser MW, Ohlbauer M, Rieder J, et al. Critical care management of major hydro- fluoric acid burns: a case report, review of the literature, and recommendations for therapy. Burns. 2004;30(4):391-398.
6. Takase I, Kono K, Tamura A, et al. Fatality due to acute fluoride poisoning in the workplace. Leg Med. 2004;6(3):197-200.

7. Burke WJ, Hoegg UR, Phillips RE. Systemic fluoride poisoning resulting from a fluoride skin burn. J Occup Med. 1973;15(1):39-41.

8. Murao M. Studies on the treatment of hydrofluoric acid burn. Bull Osaka Med Coll. 1989;35(1-2):39-48.

9. Alexeeff GV, Lewis DC, Ragle NL. Estimation of potential health effects from acute exposure to hydrogen fluoride using a "benchmark dose" approach. Risk Anal. 1993;13(1):63-69.

10. Lund K, Ekstrand J, Boe J, et al. Exposure to hydrogen fluoride: an experimental study in humans of concentrations of fluoride in plasma, symptoms, and lung function. Occup Environ Med. 1997;54(1):32-37.

11. Dalamaga M, Karmaniolas K, Nikolaidou A, et al. Hypocalcemia, hypomagnesemia, and hypokalemia following hydrofluoric acid chemical injury. J Burn Care Res. 2008;29(3):541-543.

12. Alhazzani W, Alshahrani M, Jaeschke R, et al. Neuromuscular blocking agents in acute respiratory distress syndrome: a systematic review and meta-analysis of randomized controlled trials. Crit Care. 2013;17(2):R43. 